\title{
Profile of polio-compatible cases in Nigeria, 2006-2016
}

\author{
Abdullahi Walla Hamisu", Faisal Shuaib², Ticha Muluh Johnson', Kehinde Craig ${ }^{1}$, Braka Fiona', Richard Banda', \\ Sisay G. Tegegne', Ajiboye Oyetunji', Tesfaye B. Erbeto ${ }^{1}$, Peter Nsubuga ${ }^{3}$, Rui Gama Vaz ${ }^{4}$, Ado J. G. Muhamed ${ }^{2}$ \\ and Adamu Usman²
}

\begin{abstract}
Background: The tremendous progress made by Nigeria towards polio eradication has recently suffered a setback with the isolation of circulating vaccine-derived poliovirus (cVDPV) type 2 from environmental samples and confirmation of four wild poliovirus (WPV) cases from acute flaccid paralysis (AFP) cases, with dates of onset of paralysis in July and August 2016. All these viruses were confirmed from the security-challenged northeastern state of Borno. Polio-compatible cases exist in Nigeria, and they indicate surveillance failure. Surveillance, therefore, has to be strengthened for the country to achieve certification. The objective of this paper is to highlight the epidemiological profile and magnitude of polio-compatible cases in Nigeria during the reporting period, as well as immunization and surveillance response activities conducted to close immunity and surveillance gaps.
\end{abstract}

Methods: We conducted a retrospective review of AFP surveillance performance and polio-compatible cases in Nigeria between 2006 and 2016 from the AFP database at the World Health Organization Country Office. We also reviewed and compared key epidemiological features of polio-compatible cases with those of wild poliovirus cases during the reporting period.

Results: The non-polio AFP rate improved from 6.5 in 2006 to 19.5 in 2016. The corresponding figures for stool adequacy rates were 88 and $98 \%$. The total number of polio-compatible cases reported during the reporting period was 888 , with the highest number (194) of cases reported in 2006 and the least (24) in 2016. Clusters of polio-compatible cases were reported every year during the reporting period except in 2015. The highest number (65) of polio-compatible cases in clusters was reported in 2006. The key epidemiological features of polio-compatible and wild poliovirus cases were similar.

Conclusion: AFP surveillance performance has improved significantly during the reporting period. Surveillance gaps still existed as shown by the presence of orphan viruses and polio-compatible cases, and these gaps need to be identified and closed to achieve certification.

Keywords: AFP surveillance, Polio-compatible, Wild poliovirus

\section{Background}

The tremendous progress made by Nigeria towards polio eradication, especially in the last 2 years from 2014, suffered a setback in the second quarter of 2016. A circulating vaccine-derived poliovirus (cVDPV) type 2 was isolated from the environmental samples collected in March 2016 and four wild polioviruses (WPVs) from acute flaccid paralysis (AFP) cases with dates of onset

\footnotetext{
* Correspondence: abdullahih@who.int

${ }^{1}$ World Health Organization, Country Representative Office, Abuja, Nigeria Full list of author information is available at the end of the article
}

of paralysis in July and August 2016 were confirmed. All these viruses were confirmed from the security-challenged northeastern state of Borno [1, 2].

For polio eradication, AFP surveillance is needed to identify possible areas of poliovirus transmission or cases of importation. Surveillance is also critical for documenting the absence of poliovirus circulation for polio-free certification [3]. A country's surveillance system should be sensitive enough to detect at least one case of AFP for every 100,000 children under the age of 15 years, even in the absence of polio [4]. In the case of polio endemic countries, however, 
at least two cases of AFP for every 100,000 children under the age of 15 years are required [5].

In Nigeria, the AFP surveillance performance as gauged by the non-polio AFP and stool adequacy rates has been impressive during the period from 2006 to 2016 [6]. In 2016, for instance, the country recorded a non-polio AFP rate of 19.5 cases per 100,000 children $<15$ years old (the target is 2.0/100,000). The stool adequacy rate during the same period was $98 \%$ (the target is $80 \%)$. Despite this performance, however, surveillance gaps, especially at subnational levels, exist as shown by the presence of polio-compatible cases.

Polio-compatible cases are those AFP cases for whom two adequate stool specimens were not collected, have negative laboratory results, 60-day follow-up reveals residual paralysis, and patients are lost to follow-up or died before follow-up. Such cases are referred to an expert committee which classifies them as compatible based on clinical, epidemiological, and laboratory evidence.

Additional evidence of surveillance gaps includes findings from rapid surveillance assessments conducted within the reporting period which showed knowledge gaps among key surveillance personnel, missed AFP cases, and inadequate active surveillance and documentation [7-10].

The occurrence of polio-compatible cases indicates surveillance failure and therefore the system may not be fully relied upon to exclude with certainty the existence of areas of poliovirus transmission. Similarly, such compatible cases should be monitored for clustering in space and time. Clustering occurs when two or more polio-compatible cases occur in any Local Government Area (LGA) with the date of onset of paralysis within 2 months of each other $[11,12]$.

Nigeria must strive to maintain a very sensitive polio surveillance system in order to achieve certification. All deficiencies in the surveillance system must be identified and addressed to ensure timely poliovirus detection and response. This was the path followed by other countries that have attained a polio-free status [13].

This study describes the performance of AFP surveillance and shows the key epidemiological features of polio-compatible cases and how these compare with those of WPV cases during the period between 2006 and 2016.

\section{Methods}

\section{Study design, area, and population}

We conducted a retrospective review of routinely collected data on AFP, WPV, and polio-compatible cases in Nigeria between 2006 and 2016.

\section{Description of the AFP surveillance system in Nigeria}

The AFP surveillance system in Nigeria is both health facility- and community-based. There is a network of prioritized reporting sites (both public and private health facilities) and community informants comprising patent medicine vendors, traditional and spiritual healers, traditional bone setters, and traditional birth attendants spread across all the political wards, states, and LGAs in the country.

The health facility focal person in a reporting site, as well as other health workers or clinicians, detect and report AFP cases to the LGA Disease Surveillance and Notification Officer (DSNO). The DSNO, in turn, has the responsibility of investigating the reported cases including stool specimen collection and transportation to the national polio laboratory under reverse cold chain conditions. The LGA DSNO is supported by an assistant and World Health organization (WHO) LGA facilitator. Community informants refer AFP cases to the nearest reporting site or report cases directly to the LGA DSNO. Active surveillance at the LGA level is conducted by the LGA DSNO, their assistant, WHO LGA facilitators, and field volunteers.

At the state level, the state epidemiologist and the state DSNO coordinate surveillance activities including organizing monthly LGA DSNO meetings, outbreak investigation, supervision, training, and sensitization activities. The WHO cluster consultants support the state and LGA surveillance focal points, conduct active surveillance, verify reported AFP cases, and conduct 60-day follow-up of inadequate AFP cases, confirmed poliovirus cases, and cases with Sabin (i.e., vaccine) virus.

At the national level, surveillance is coordinated by the national epidemiologist who receives and analyses laboratory results from the two national polio laboratories and shares feedback with stakeholders, conducts supervision and monitors surveillance performance, and organizes surveillance assessments, outbreak investigation, peer reviews, and training, and supports the polio laboratories and the national polio committees. WHO supports the surveillance system at all levels including the provision of logistics support to the DSNOs, national polio laboratories, and the national polio committees.

There is a five-member National Polio Expert Committee (NPEC) which meets quarterly to classify AFP cases brought to it by the secretariat. The secretariat is provided by the National Primary Health Care Development Agency (NPHCDA) and supported by WHO.

\section{Data collection and analysis}

We extracted data on the two core AFP surveillance performance indicators of non-polio AFP and stool adequacy rates. We specifically extracted data on age, sex, and doses of oral polio vaccine received for poliocompatible and WPV cases. We also identified the trend, number of states, and LGAs with clustering of polio-compatible cases. 


\section{Results}

The surveillance system in Nigeria is both health facility and community-based (Fig. 1). The non-polio AFP rate improved from 6.5 in 2006 to 19.5 in 2016 . The corresponding figures for stool adequacy rates were 88 and 98\% (Fig. 2).

During the reporting period, the total number of polio-compatible cases reported was 888 out of which 516 (58\%) were males and $372(42 \%)$ females. Most $(82 \%)$ of the compatible cases were under the age of 5 years. A total of 137 (15\%) of the compatible cases did not receive any oral polio vaccine (OPV), while 255 (29\%) received 1-3 OPV doses, and 496 (56\%) received 3+ OPV doses (Table 1).

During the reporting period, there was a cluster of 235 polio-compatible cases. The highest number of states affected was in 2006 and 2011 with 11 and 10 states, respectively. The highest number of clusters (65 and 45) were also recorded in 2006 and 2011, respectively, and the corresponding number of affected LGAs were 26 and 19 in the same years (Table 2).

Of the $2861 \mathrm{WPV}$ cases reported during the reporting period, 1638 (57\%) were males and 1223 (43\%) females. A total of $843(30 \%)$ of WPV cases did not receive OPV, while 1438 (50\%) received 1-3 OPV doses, and 576 (20\%) received 3+ OPV doses (Table 3).

\section{Discussion}

We found that the AFP surveillance performance in the country has consistently improved from 2006 to 2016 with the highest level of performance in 2016 which was above the minimum requirement of WHO in each of those years [14]. The presence of polio-compatible cases, however, indicates the existence of surveillance gaps through failure to collect adequate stool specimens, and this is more so if there is clustering of compatible cases [15].

In Nigeria, clustering of polio-compatible cases occurred in each of the years during the reporting period, except in 2015 . The country witnessed the highest number of clustering of compatible cases in 2006 and 2011, representing periods with particularly serious surveillance gaps and, despite the high number of WPV cases reported during these years, many more cases could have been missed as a result of failure to timely detect AFP cases. However, the consistent decline in the number of poliocompatible cases from 194 in 2006 to 24 in 2016 as well as the declining clustering of cases shows much-improved surveillance in the country. Surveillance gaps at this stage of polio eradication are a global threat and must be adequately addressed [16]. The occurrence of polio-compatible cases is a global phenomenon [17], and cases have been known to occur in countries with sensitive AFP surveillance [18-20].

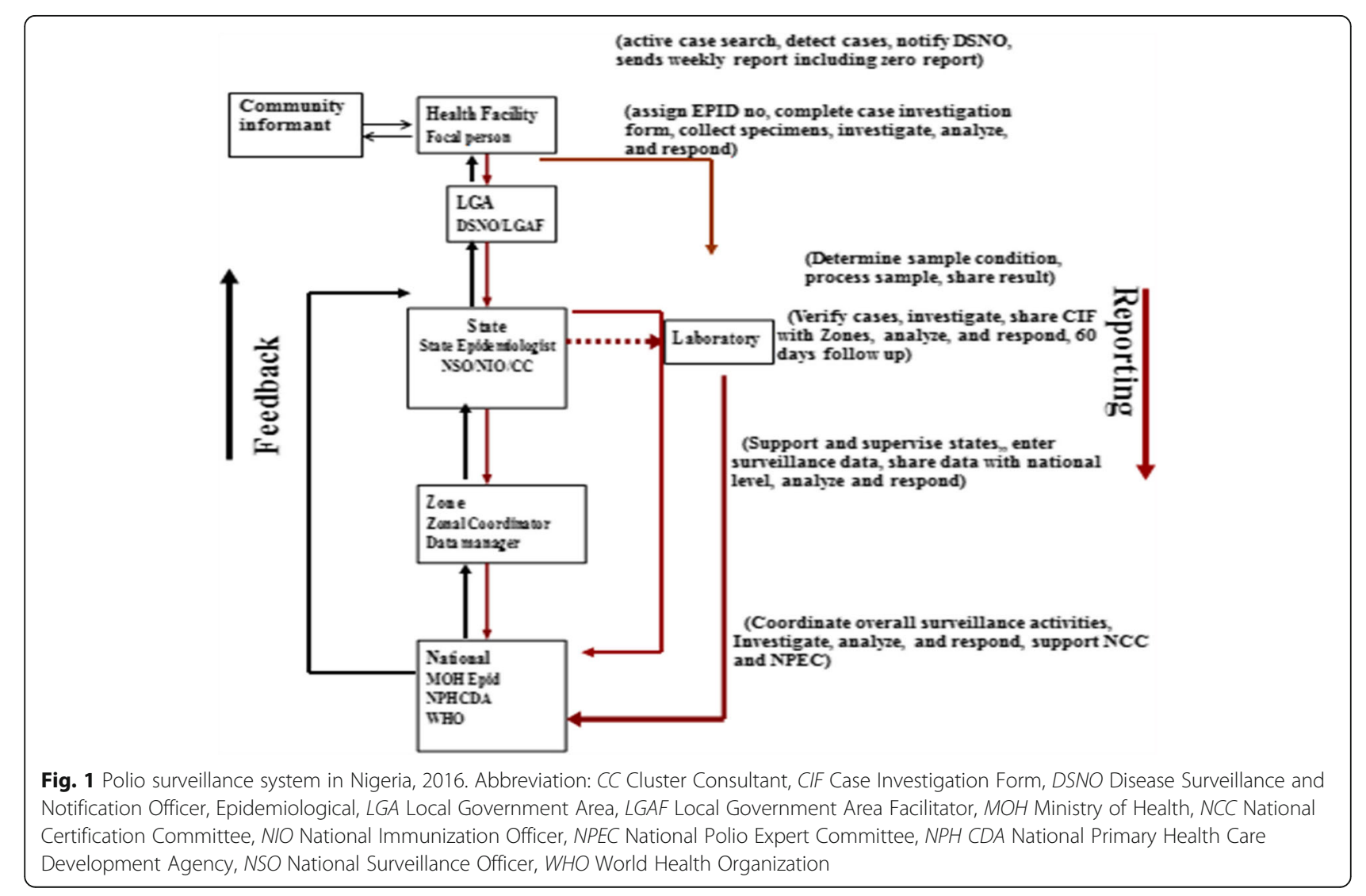


Acute Flaccid Paralysis Surveillance Performance in Nigeria (2006-2016)

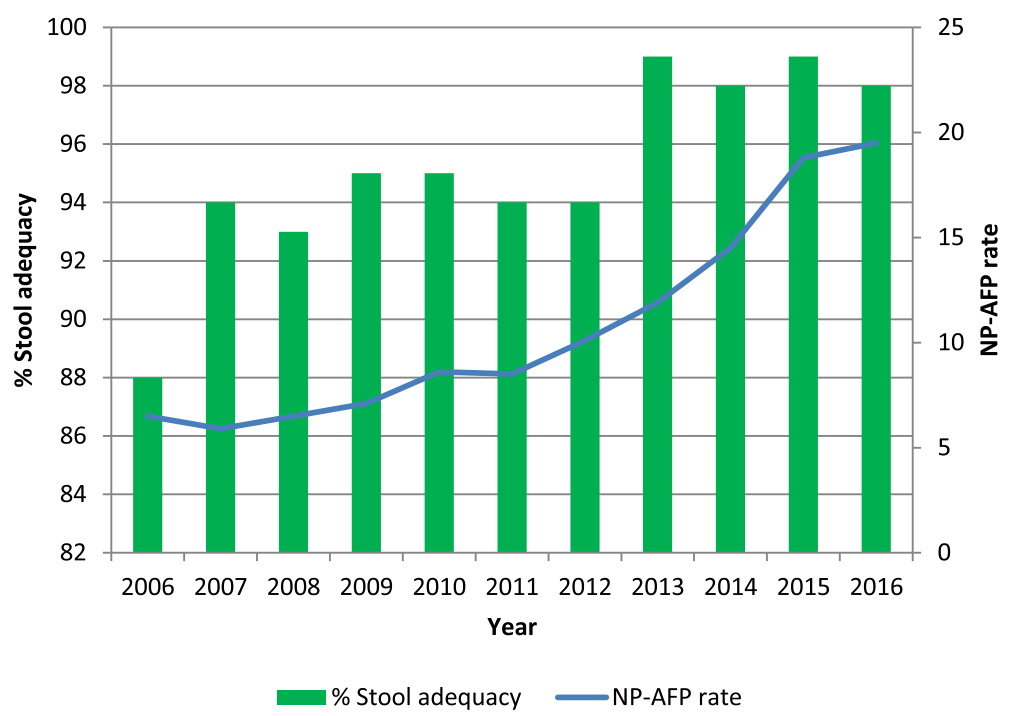

Fig. 2 Acute flaccid paralysis surveillance performance in Nigeria (2006-2016). NP-AFP non-polio acute flaccid paralysis

The AFP surveillance system in Nigeria has a definite protocol for responding to polio- compatible cases. This response has both immunization and surveillance components. During field investigation of a compatible case, OPV is administered to those under 5 years of age in the vicinity (or settlement) of the case to serve as an immediate immunization response. The main immunization response, however, is that three rounds of immunization campaigns should be conducted with bivalent OPV (bOPV) targeting the age group of $0-59$ months in the index ward with contiguous concentric wards depending on risk analysis using several datasets (routine immunization, supplemental immunization activities, mop up, and surveillance). The immunization response follows the following guide: once a case is classified as compatible, the number of supplemental immunization activities in the area since the date of onset of paralysis is determined. If supplemental immunization activities were conducted less than 1 month from onset of paralysis, then that immunization activity is considered to be a round-one response. Two immunization response activities should then be conducted 2 to 3 weeks apart. If no supplemental immunization activity was conducted less than 1 month from onset of paralysis, then three rounds of immunization activities will be conducted. If three or more rounds of immunization activities have been conducted from the date of onset of paralysis to

Table 1 Trend and key epidemiological features of polio-compatible cases in Nigeria, 2006-2016

\begin{tabular}{|c|c|c|c|c|c|c|c|c|c|c|}
\hline \multirow[t]{2}{*}{ Year } & \multirow{2}{*}{$\begin{array}{l}\text { Number of } \\
\text { compatible } \\
\text { cases }\end{array}$} & \multicolumn{2}{|l|}{ Sex } & \multicolumn{4}{|c|}{ Age (months) } & \multicolumn{3}{|c|}{ Oral polio vaccine doses } \\
\hline & & $\begin{array}{l}\text { Male } \\
n(\%)\end{array}$ & $\begin{array}{l}\text { Female } \\
n(\%)\end{array}$ & $\begin{array}{l}<12 \\
n(\%) \\
\end{array}$ & $\begin{array}{l}12-35 \\
n(\%) \\
\end{array}$ & $\begin{array}{l}36-59 \\
n(\%)\end{array}$ & $\begin{array}{l}60+ \\
n(\%)\end{array}$ & $\begin{array}{l}0 \\
n(\%)\end{array}$ & $\begin{array}{l}1-3 \\
n(\%)\end{array}$ & $\begin{array}{l}3+ \\
n(\%)\end{array}$ \\
\hline 2006 & 194 & $110(57)$ & $84(43)$ & $18(9)$ & $129(67)$ & $33(17)$ & $14(7)$ & $51(26)$ & $90(46)$ & $53(28)$ \\
\hline 2007 & 74 & $41(55)$ & $33(45)$ & $5(7)$ & $46(62)$ & $13(18)$ & $10(13)$ & $22(30)$ & $31(42)$ & $21(28)$ \\
\hline 2008 & 93 & $53(57)$ & $40(43)$ & $10(11)$ & $52(56)$ & $19(20)$ & $12(13)$ & $21(23)$ & $36(39)$ & $36(38)$ \\
\hline 2009 & 75 & $41(55)$ & $34(45)$ & $4(5)$ & $40(53)$ & $13(18)$ & $18(24)$ & $9(12)$ & $23(31)$ & $43(57)$ \\
\hline 2010 & 79 & $44(56)$ & $35(44)$ & $8(10)$ & $36(46)$ & $20(25)$ & $15(19)$ & $9(11)$ & $27(34)$ & $43(55)$ \\
\hline 2011 & 141 & $93(66)$ & $48(34)$ & $8(6)$ & $59(42)$ & $42(30)$ & $32(22)$ & $14(10)$ & $25(18)$ & $102(72)$ \\
\hline 2012 & 67 & $38(57)$ & $29(43)$ & $3(4)$ & $32(48)$ & $16(24)$ & $16(24)$ & $5(7)$ & $12(18)$ & $50(75)$ \\
\hline 2013 & 81 & $45(56)$ & $36(44)$ & $5(6)$ & $36(45)$ & $13(16)$ & $27(33)$ & $5(6)$ & $7(9)$ & $69(85)$ \\
\hline 2014 & 35 & $20(57)$ & $15(43)$ & $3(9)$ & $19(54)$ & $7(20)$ & $6(17)$ & 1 (3) & 1 (3) & 33 (94) \\
\hline 2015 & 25 & $16(63)$ & $9(37)$ & $3(12)$ & $16(67)$ & $2(9)$ & $3(12)$ & $0(0)$ & $3(8)$ & $22(92)$ \\
\hline 2016 & 24 & $15(63)$ & $9(37)$ & $3(13)$ & $13(54)$ & $5(12)$ & $3(12)$ & $0(0)$ & $0(0)$ & $24(100)$ \\
\hline Overall & 888 & $516(58)$ & $372(42)$ & $70(8)$ & $465(54)$ & $182(20)$ & $156(18)$ & 137 (15) & $255(29)$ & $496(56)$ \\
\hline
\end{tabular}


Table 2 Trend and distribution of compatible clusters in states and Local Government Areas (LGAs) in Nigeria, 2006-2016

\begin{tabular}{llll}
\hline \multirow{2}{*}{ Year } & $\begin{array}{l}\text { Compatible clusters } \\
\text { Number of } \\
\text { compatible cases }\end{array}$ & $\begin{array}{l}\text { Number } \\
\text { of states }\end{array}$ & $\begin{array}{l}\text { Number } \\
\text { of LGAs }\end{array}$ \\
\hline 2006 & 65 & 11 & 26 \\
2007 & 21 & 3 & 9 \\
2008 & 28 & 7 & 11 \\
2009 & 14 & 5 & 6 \\
2010 & 22 & 7 & 10 \\
2011 & 45 & 10 & 19 \\
2012 & 17 & 6 & 8 \\
2013 & 16 & 5 & 6 \\
2014 & 4 & 2 & 2 \\
2015 & 0 & 0 & 0 \\
2016 & 3 & 1 & 1 \\
Overall & 235 & 56 & 97 \\
\hline
\end{tabular}

the date of classification of the case in the locality, then there is no need for any immunization response.

For the surveillance response upon notification of a compatible case, the outbreak investigation team will conduct an investigation within $48 \mathrm{~h}$, including a search for active cases in the affected community and in all health facilities in the affected ward and neighboring wards within 1 week of case notification. During the mop-up immunization activities, the DSNO provides training to vaccinators on case finding and reporting. In the Borno state, which is experiencing security challenges due to the insurgent Boko Haram, extra measures were taken to ensure quality immunization and surveillance responses through partnership with the military and civilian security organizations. Security personnel accompany vaccination teams during immunization campaigns in inaccessible and partially accessible areas and also support investigation of AFP cases. In addition, special teams were formed to reach high-risk populations in the Internally Displaced Persons' camps and host communities.

Polio-compatible cases are not true polio cases but are classified as such since the occurrence of polio in such cases cannot be ruled out [21]. The occurrence of polio-compatible cases is, however, not inevitable since the final classification is largely dependent on the information available to the NPEC. Many countries, including Algeria and Rwanda, did not report any polio-compatible case during the reporting period [22]. Polio-compatible cases should be investigated to identify the underlying cause for effective intervention [23]. In addition to showing areas of surveillance gaps, compatible polio cases also influence the choice of areas to be targeted for mop-up campaigns [24].

The age distribution and sex ratio as well as geographic spread of polio-compatible and WPV cases were similar to the cases in the Democratic Republic of the Congo [25]. Male preponderance has, however, been noted in both wild and polio-compatible data during the study period as was noted in earlier studies, and this may be due to more parental concern about their male children [26].

We found that most (>90\%) inadequate AFP cases were due to late detection. Late detection and notification are often due to ignorance and poor perception of disease epidemiology by caregivers who seek alternative health care as their first choice because of the belief that paralysis symptoms are spiritually induced [27].

One of the key limitations of this paper is that data on the OPV received by both polio-compatible and WPV cases were historical and not based on records such as immunization cards. Such historical data may be subject

Table 3 Trend and key epidemiological features of wild poliovirus (WPV) cases in Nigeria, 2006-2016

\begin{tabular}{|c|c|c|c|c|c|c|c|c|c|c|}
\hline \multirow[t]{2}{*}{ Year } & \multirow{2}{*}{$\begin{array}{l}\text { Number } \\
\text { of WPVs }\end{array}$} & \multicolumn{2}{|l|}{ Sex } & \multicolumn{4}{|c|}{ Age (months) } & \multicolumn{3}{|c|}{ Oral polio vaccine doses } \\
\hline & & $\begin{array}{l}\text { Male } \\
n(\%)\end{array}$ & $\begin{array}{l}\text { Female } \\
n(\%)\end{array}$ & $\begin{array}{l}<<12 \\
n(\%)\end{array}$ & $\begin{array}{l}12-35 \\
n(\%)\end{array}$ & $\begin{array}{l}36-59 \\
n(\%)\end{array}$ & $\begin{array}{l}60+ \\
n(\%)\end{array}$ & $\begin{array}{l}0 \\
n(\%)\end{array}$ & $\begin{array}{l}1-3 \\
n(\%)\end{array}$ & $\begin{array}{l}3+ \\
n(\%)\end{array}$ \\
\hline 2006 & 1122 & $649(58)$ & $473(42)$ & $82(7)$ & $767(68)$ & $219(20)$ & $54(5)$ & $412(37)$ & $558(50)$ & $152(14)$ \\
\hline 2007 & 286 & $163(57)$ & $123(43)$ & $24(8)$ & $183(64)$ & $66(23)$ & $13(5)$ & $73(26)$ & $155(54)$ & $58(20)$ \\
\hline 2008 & 796 & $452(57)$ & $344(43)$ & $43(5)$ & $544(68)$ & $181(23)$ & $28(4)$ & $235(30)$ & $428(54)$ & $133(17)$ \\
\hline 2009 & 388 & $225(58)$ & $163(42)$ & $25(6)$ & $237(61)$ & $94(24)$ & $32(8)$ & $66(17)$ & $197(51)$ & $125(32)$ \\
\hline 2010 & 21 & $17(81)$ & $4(19)$ & $0(0)$ & $15(71)$ & $4(19)$ & $2(10)$ & $3(14)$ & 7 (33) & $11(52)$ \\
\hline 2011 & 63 & $40(63)$ & $23(37)$ & $3(5)$ & 39 (62) & $14(22)$ & $7(11)$ & $20(32)$ & $22(35)$ & $21(33)$ \\
\hline 2012 & 122 & $64(52)$ & $58(48)$ & $11(9)$ & $75(61)$ & $29(24)$ & $7(6)$ & $27(22)$ & $44(36)$ & $51(42)$ \\
\hline 2013 & 53 & $24(45)$ & $29(55)$ & $4(8)$ & $30(57)$ & $12(23)$ & $7(13)$ & $7(13)$ & $21(40)$ & $25(47)$ \\
\hline 2014 & 6 & $3(50)$ & $3(50)$ & $0(0)$ & $4(67)$ & $1(17)$ & $1(17)$ & $0(0)$ & $6(100)$ & $0(0)$ \\
\hline 2015 & 0 & $0(0)$ & $0(0)$ & $0(0)$ & $0(0)$ & $0(0)$ & $0(0)$ & $0(0)$ & $0(0)$ & $0(0)$ \\
\hline 2016 & 4 & $1(25)$ & $3(75)$ & $0(0)$ & $3(75)$ & $1(25)$ & $0(0)$ & $0(0)$ & $0(0)$ & $0(0)$ \\
\hline Overall & 2861 & $1638(57)$ & $1223(43)$ & $192(7)$ & $1897(66)$ & $621(22)$ & $151(5)$ & $843(30)$ & $1438(50)$ & $576(20)$ \\
\hline
\end{tabular}


to bias, including that of recall. This limitation may have accounted for the significant difference in the proportion of OPV "zero dose" in the two groups. Also, OPV doses received during routine and supplemental immunization activities were not differentiated, making it difficult to comment on the strength or otherwise of these forms of immunization strategies. Another limitation is the incomplete records of the detailed investigation of compatible and WPV cases to enable comparison of other epidemiological features such as environmental settings, socioeconomic status of caregivers, and travel history.

Delayed AFP detection may lead to late polio outbreak detection and response which, in turn, hinders progress towards eradication and substantially raises the cost of the eradication program [28]. Furthermore, late outbreak detection has led to re-established transmission in many hitherto polio-free countries [29].

\section{Conclusion}

We conclude that AFP surveillance performance in the country has improved significantly during the reporting period. Surveillance gaps, however, still exist as evidenced by the presence of polio-compatible cases, and these gaps need to be identified and closed to achieve certification.

We recommend that strategies leading to early AFP detection such as the conduct of active surveillance, training, and community and clinician sensitization, as well as the expansion of the reporting network should be given high priority.

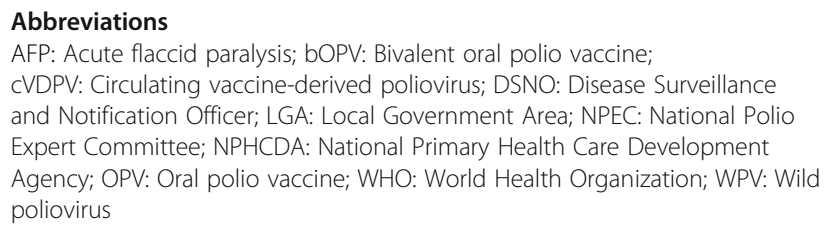

\section{Acknowledgements}

The authours are greatful to the monitoring and evaluation unit of WHO country office for their support in providing access to the database used for this publication. We are also deeply appreciative of the WHO country office, Nigeria for supporting the process and finalization of this publication.

\section{Funding}

Publication of this article was sponsored by the WHO country office, Nigeria.

\section{Availability of data and materials}

The datasets generated and/or analyzed during the current study are available in the Nigeria AFP surveillance database, resident at the WHO Country Office, Nigeria. Permission to use the data was given by the WHO Country Office through the Country Representative.

\section{About this supplement}

This article has been published as part of BMC Public Health Volume 18 Supplement 4, 2018: Experiences and lessons learned in polio eradication in Nigeria. The full contents of the supplement are available online at https:// bmcpublichealth.biomedcentral.com/articles/supplements/volume-18supplement-4.

\section{Authors' contributions}

All authors contributed to the article. AW, TM, KC, BF, RB, SG, AO, TB, and RG contributed in the concept, data analysis and discussion. PN contributed with discussions and analysis; while AG, FS and AU contributed with discussions. All of the authors have read and approved the final manuscript.

\section{Ethics approval and consent to participate}

The data used in this study were secondary to those obtained after appropriate permission from the WHO Country Office. The data are available at the WHO country server. No ethical consent or permission to participate was therefore required.

\section{Consent for publication}

Not applicable.

\section{Competing interests}

The authors declare that they have no competing interests.

\section{Publisher's Note}

Springer Nature remains neutral with regard to jurisdictional claims in published maps and institutional affiliations.

\section{Author details}

${ }^{1}$ World Health Organization, Country Representative Office, Abuja, Nigeria. ${ }^{2}$ National Primary Health Care Development Agency, Abuja, Nigeria. ${ }^{3} \mathrm{Global}$ Public Health Solutions, Atlanta, GA, USA. ${ }^{4}$ World Health Organization, Geneva, Switzerland.

Published: 13 December 2018

\section{References}

1. Bolu O, Nnadi C, Damisa E, Braka F, Siddique A, Archer WR, et al. Progress towards poliomyelitis eradication-Nigeria, January-December 2017. MMWR. 2018;67(8):253-6.

2. Gardner TJ, Diop OM, Jorba J, et al. Surveillance to track progress toward polio eradication—worldwide, 2016-2017. MMWR. 2018;67(14):418-23.

3. Tangermann RH, Lamoureux C, Tallis G, Goel A. The critical role of acute flaccid paralysis surveillance in the global polio eradication initiative. Int Health. 2017;9:156-63.

4. Odoom JK, Ntim NAA, Sarkodie B, Addo J, Kren Minta-Asare K, Obodai E, et al. Evaluation of AFP surveillance indicators in polio-free Ghana, 2009-2013. BMC Public Health. 2014;14:687.

5. Khan F, Datta SD, Quddus A, Vertefeuille JF, et al. Progress toward polio eradication—worldwide, January 2016-march 2018. MMWR. 2018;67(18): 524-8.

6. Hamisu AW, Johnson TM, Craig K, Mkanda P, Banda R, Tegegne SG, et al. Strategies for improving polio surveillance performance in the securitychallenged Nigerian states of Adamawa, Borno, and Yobe during 20092014. J Infect Dis. 2016;213(Suppl 3):136-9.

7. Hamisu AW, Johnson TM, Craig K, et al. Sensitivity of acute flaccid paralysis surveillance in Nigeria (2006-2015). J Infect Dis Treat. 2016;2(2):13.

8. Isibor I, Gasasira A, Mkanda P, Weldegriebriel G, Bassey BE, Toritseju MS, et al. Rapid assessments of acute flaccid paralysis surveillance in seven key polio high risk states in northern Nigeria. Palgo J Med Medical Sci. 2014;2(3): 33-40.

9. Saleh JEA, Vaz RG, Braka F, et al. A critical look at 2015 acute flaccid paralysis (AFP) surveillance core indicators of Bauchi state, Nigeria. Sci J Public Health. 2016;4(4):326-9.

10. Bassey E, Braka F, Ticha JM, et al. Assessment of the quality of the reverse cold chain management in the acute flaccid paralysis (AFP) surveillance system for polio eradication; south-south zone, Nigeria 2015. Am J Epidemiol Infect Dis. 2016;4(5):100-4.

11. Kohler KA, Hlady WG, Banerjee K, Gupta D, Francis P, Durrani S, et al. Compatible poliomyelitis cases in India during 2000. Bull World Health Organ. 2002;80(02):2-9.

12. Tajaldin B, Almilaji $K$, Langton $P$, et al. Defining polio: closing the gap in global surveillance. Ann Glob Health. 2015;4(4):1-10.

13. Snider CJ, Diop OM, Burns CC, et al. Surveillance systems to track progress toward polio eradication-worldwide, 2014-2015. MMWR. 2016;65(13):346-51. 
14. Andrus JK, Thapa AB, Withana N, et al. A new paradigm for international disease control: lessons learned from polio eradication in Southeast Asia. Am J Public Health. 2001;91(1):146-50.

15. Sathyamala C, Mittal O, Dasgupt R, Priya R. Polio eradication initiative in India: deconstructing the GPEl. Int J Health Serv. 2005;35(2):361-83.

16. Maes EF, Diop OM, Jorba J, et al. Surveillance systems to track progress toward polio eradication—worldwide, 2015-2016. MMWR. 2017;66(13):359-65.

17. CaseCount. https://extranet.who.int/polis/public/CaseCount.aspx. Accessed 31 Dec 2016.

18. Luo H, Zhang Y, Wang $X$, Yu W, Wen N, Yan D, et al. Identification and control of a poliomyelitis outbreak in Xinjiang, China. N Engl J Med. 2013;369:1981-90.

19. Hussain I, Ali S, Sinniah M, Kurup D, Khoo TB, Thomas TGS, et al. Five-year surveillance of acute flaccid paralysis in Malaysia. J Paediatr Child Health. 2004:2003:127-30

20. Khuzwayo LS, Kuonza LR, Ngcobo NJ. Evaluating the acute flaccid paralysis surveillance system in South Africa, 2005-2009-an analysis of secondary data. Pan Afr Med J. 2013;8688:2005-9.

21. World Health Organization. Progress towards poliomyelitis eradication. Angola, January 1998-June 2002. Wkly Epidemiol Rec 2002;77(35):290-4

22. Okeibunor JC, Ota MC, Akanmori BD, Gumede N, et al. Polio eradication in the African region on course despite public health. Vaccine. 2015;35(9): $1202-6$.

23. Blake IM, Chenoweth $\mathrm{P}$, Okayasu H, Donnelly CA, et al. Faster detection of poliomyelitis outbreaks to support polio eradication. Emerg Infect Dis. 2016;22(3):449-56

24. Hoekstra EJ, Chai F, Wang XJ, Zhang WL, Yu JJ, Bilous J. Excluding polio in areas of inadequate surveillance in the final stages of eradication in China. Bull World Health Organ. 2000;78(3):315-20,

25. Alleman MM, Meyer SA, Mulumba A, Nyembwe M, Riziki Y, Mbule A, et al. Improved acute flaccid paralysis surveillance performance in the Democratic Republic of the Congo, 2010-2012. J Infect Dis. 2014;210(Suppl 1):2010-2.

26. Rasul CH, Das PL, Phil M, Ahmed S, Ahmed M, et al. Clinical profile of acute flaccid paralysis. Med J Malaysia. 2002;57(1):61-5.

27. Macama A, Okeibunor J, Grando S, Djibaoui K, Yameogo RK, Gasasira N, Mbaye $S$, Mihigo R, et al. Reasons and circumstances for the late notification of acute flaccid paralysis (AFP) cases in health facilities in Luanda. Pan Afr Med J. 2014;8688:1-8.

28. Mbaeyi C, Mohamed A, Owino BO, Mengistu KF, et al. Strengthening acute flaccid paralysis surveillance through the village polio volunteers program in Somalia. Clin Infect Dis. 2018. https://doi.org/10.1093/cid/ciy180.

29. Wassilak S, Orenstein W. Challenges faced by the global polio eradication initiative. Vaccine. 2010;9(5):447-9.

Ready to submit your research? Choose BMC and benefit from:

- fast, convenient online submission

- thorough peer review by experienced researchers in your field

- rapid publication on acceptance

- support for research data, including large and complex data types

- gold Open Access which fosters wider collaboration and increased citations

- maximum visibility for your research: over $100 \mathrm{M}$ website views per year

At $\mathrm{BMC}$, research is always in progress.

Learn more biomedcentral.com/submissions 